\title{
What About a Reductionist Approach? Comments on Terry Horgan
}

\author{
Christian Sachse
}

Received: 30 September 2006/ Accepted: 31 May 2007/Published online: 6 September 2007

(C) Springer Science+Business Media B.V. 2007

\begin{abstract}
In his work, Horgan argues for the compatibilism of agency, mental state-causation, and physical causal-closure. We generally assume a causally closed physical world that seems to exclude agency in the sense of mental state-causation in addition to physical causation. However, Horgan argues for an account of agency that satisfies the experience of our own as acting persons and that is compatible with physical causal-closure. Mental properties are causal properties but not identical with physical properties because there are different ontological levels. In this commentary, I shall reconsider the essential issues of this compatibilism (1), focus on a problem for Horgan's conception of agent causation that arises from the causal argument for ontological reductionism (2), and propose to embed Horgan's conception of agency within a reductionist approach in order to vindicate the indispensable character of agency (3).
\end{abstract}

\section{Horgan's Compatibilism}

Horgan considers the relationship between agentive experience, mental statecausation and physical causal-closure. Thereby, he claims the compatibility of agentive experience with both mental state-causation and physical causal-closure. Let me briefly sum up the mentioned issues in order to express a common ground.

Mental state-causation means that the behaviour we classify as genuine actions is caused by certain mental states such as decisions, beliefs, desires, etc. (cf. Horgan's abstract). Agentive experience is a certain experience of oneself whenever one acts intentionally. This agentive experience includes a "specific purpose" and a "voluntariness" of one's own actions (cf. Horgan's first section). Physical causalclosure means that the "state of the world at any moment in time, insofar as it is

C. Sachse $(\square)$

University of Lausanne, Lausanne, Switzerland

e-mail: christian.sachse@unil.ch 
diachronically determined at all, is diachronically determined by prior physics-level phenomena, on basis of the fundamental laws of physics" (cf. Horgan's second section). Against this background, Horgan raises the question whether physical causal-closure excludes mental state-causation and agency.

In order to argue for these hypotheses being compatible with one another, Horgan begins with a reconsideration of agency. A vindication of agency requires both the argument for mental state-causation and the compatibility of mental state-causation with genuine agency (cf. Horgan's third section). In this work, Horgan focuses on the compatibility of mental state-causation with physical causal-closure (the compatibility of mental state-causation and agency will be considered in two forthcoming papers of Horgan).

The problem for such a compatibilism can be summed up as follows: Since on the one hand, the physical domain is causally closed and, on the other hand, mental property tokens are causal property tokens, it seems to be excluded that mental property tokens are causal ones in addition to configurations of physical property tokens. This exclusion problem is considered in detail by Kim (2005, chap. 2). How can we thus maintain that mental property tokens are not identical with configurations of physical property tokens? Horgan argues against such a tokenidentity in terms of explanations: a causal explanation in mental terms is compatible with a causal explanation in physical terms because these explanations are situated at different levels of explanation. There are different true and objective patterns of counterfactual dependence that do not exclude each other. On this basis, Horgan concludes that there are different ontological levels. For instance, the causal patterns from tokens of agency to tokens of certain behaviour are at a different ontological level compared to the causal patterns that physics considers.

\section{Ontological Problems for Horgan's Compatibilism}

Horgan's contextualist approach in terms of different ontological levels does not avoid the exclusion problem. My critique of Horgan's compatibilism proceeds as follows: First of all, Horgan agrees with the principle of physical causal-closure (Sect. 4, numbered statement no. 1). Thus, for any physical property token, say $p_{2}$, insofar as $p_{2}$ has a cause, it has a complete physical cause, say $p_{1}$. Secondly, he rejects ontological reductionism (Sect. 4, numbered statements no. 2-4). In summary, mental property tokens are not identical with physical property tokens. Say, for instance, the mental property token $m_{1}$ is not identical with its physical supervenience base $p_{1}$. Finally, Horgan defends mental to physical causation. This means that mental property tokens often have physical effects- $m_{1}$ causes $p_{2}$ for instance. As a result of this, either the physical causal-closure is false, or there is systematic overdetermination. Either $p_{1}$ is not the complete cause of $p_{2}$ such that the causal-closure of the physical domain is false, or, $p_{2}$ has two sufficient causes $-m_{1}$ and $p_{1}$.

Horgan's approach of contextualisation does not avoid this result. That there are two levels of explanation-the mental and the physical one-does not imply two different ontological levels, as Heil points out (2003, chap. 3). From an ontological 
point of view, physical causes are sufficient for any change within the physical state of the world. Thus, the non-identity of the mental with something physical and the mental to physical causation lead to systematic overdetermination. If, on the other hand, physical causes were not sufficient for every change within the physical domain, the physical causal-closure would be false. Leaving aside systematic overdetermination, Horgan's proposed strategy does not lead to an anti-reductionist compatibilism of mental state-causation and physical causal-closure.

Therefore, taking physical causal-closure and mental state-causation for granted, ontological reductionism seems to be the only option. In order to avoid epiphenomenalism with regard to the mental, and in order not to contradict physical causal-closure (or to claim systematic overdetermination), the identity of mental property tokens with configurations of physical property tokens is wellargued. All there is in the world is something physical. In this context, Horgan's compatibilism fails to be an ontological alternative to ontological reductionism. There is no ontological difference between mental property tokens and certain complex configurations of physical property tokens because they have the same effects. In this context, the special sciences and physics describe the same entities, that is, the same configurations of property tokens. Against this background, I shall consider Horgan's contextualist approach and compatibilism with regard to theories and concepts. What is the relationship between different sciences and descriptions if they describe and explain one and the same entities?

\section{Compatibililism of Horgan's Approach with Epistemological Reductionism}

First of all, let me reconsider Horgan's compatibilism. Horgan's approach claims that an antireductionist position is compatible with the causal closure of physics. The fact that there are different levels of explanations (different counterfactual dependencies) is, in the last resort, an argument in favour of there being different ontological levels. However, as outlined in the previous section, there is a strong argument in favour of ontological reductionism. Thus, any token of an agentive experience of the behaviour of oneself such as the intentional action to calculate angles is identical with a certain configuration of physical property tokens. I shall leave aside epiphenomenalism or overdetermination at this point. Therefore, one may be inclined to modify Horgan's position in the following manner: there are two descriptions of the agentive experience in question-one description in mental terms and another description in physical terms.

Let me note that, because of multiple realization, mental property types are not identical with types of configurations of physical property tokens even if any mental property token is identical with a configuration of physical property tokens. ${ }^{1}$ To put it another way, one and the same mental description type is made true by entities that make true physical descriptions of different types. However, the physical causal closure implies the following point: even if the concepts of the special sciences are generally not co-extensional with physical concepts (because of multiple

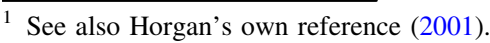


realization), any property token of the special sciences can in principle be sufficiently explained in terms of physics, which provides the most detailed causal explanations. After all, Horgan agrees with the causal closure of the physical.

Let me recap the most important issues of this commentary in the context of the current debate on reductionism in order to outline another problem for Horgan's contextualist approach. Any mental property token can in principle be reductively explained in the last resort in terms of physics (cf. Kim 2005, chap. 4; Chalmers 1996, pp. 42-51), but since mental concepts are not co-extensional with physical concepts, epistemological reductionism seems not to be feasible. This is the antireductionist suggestion of the possibility of multiple realization. However, if the mental concepts cannot be systematically connected with physical concepts, it is not intelligible how the mental concepts can bring out salient causal relations among property tokens in a way that physics cannot bring out. Anti-reductionism based on the multiple realization argument therefore ends up in eliminativism with respect to the special sciences (cf. Bickle 1998, especially chaps. 2-4). Theory reduction needs co-extensional concepts of physics and the special sciences, and, therefore, biconditional bridge-principles (cf. Endicott 1998). To conclude, a vindication of the scientific quality of concepts about agency hence seems to be only possible if these concepts are bi-conditionally connected with physical concepts. However, if a concept of the special sciences is bi-conditionally connected with a physical concept, the scientific quality of the former concept is vindicated, but it is dispensable. This is a dilemma any position faces. Does Horgan's contextualist approach help?

In his fourth section, Horgan characterises presentational intentional content as "the kind that accrues to phenomenology directly-apart from whether or not one has the capacity to articulate this content linguistically and understand what one is thus articulating". He contrasts this with "the kind of content possessed by such linguistic articulations, and by the judgments they articulate"-the judgmental intentional content. The clue of this distinction is that "the satisfaction conditions for presentational agentive intentional content-i.e. for agentive phenomenologycoincide with certain not-limit case, compatibilist, satisfaction conditions for judgmental agentive intentional content" (italics in the original). To put it another way, the presentational intentional content is compatible with the physical causal closure and mental state causation, while the judgmental intentional content is not. The satisfaction conditions of agentive phenomenology (presentational intentional content) do not require the falsity of the physical causal closure and mental state causation. However, as Horgan points out, there is a tendency to reject such a compatibilism - a mistaken tendency that is based on the failure to distinguish between presentational and judgmental intentional content: we always tend to use contextually variable implicit parameters governing the judgmental concept of freedom that are in fact not compatible with physical causal closure or state causation. Thus, even if we inquire into the presentational content of agentive phenomenology, we must not use the implicit parameters of judgmental intentional content. To conclude, each concept (e.g. of cause, agency, etc.) is governed by variable implicit semantic parameters, and the judgmental intentional content of 
agency and causation does not affect the presentational content of agentive experience.

Let me, however, insist on the following issue: either the referents of the concepts with different implicit semantic parameters are identical, or they are distinct. Against the background of the second section of this commentary, there is a strong argument for token-identity. Taking the threat of eliminativism with respect to the special sciences seriously, Horgan's approach is not sufficient to explain the following point: what is the systematic link between presentational and judgmental intentional content such that the judgmental intentional content is scientifically explanatory? As far as I can see, the challenge for the philosophy of science is not to argue for anti-reductionist approaches to the concept of agency in order to vindicate its indispensable character in explanations, but to establish a reductionist approach that is compatible with the scientific indispensable character of the concept of agency. To conclude, in order to justify the scientific quality of the concept of agency, it has to be systematically linked with, in the last resort, physics that is causally closed. A general reductionist strategy that proposes such a systematic link that takes into account multiple realization is outlined in detail in Sachse (2007, chap. 2). This reductionist approach is not eliminativist, but conservative because it takes multiple realization not as an anti-reductionist argument, but employs it only in order to justify the indispensable scientific quality of abstract concepts (cf. Esfeld and Sachse 2007). Therefore, the reduction of "agency" does not necessarily mean its elimination or replacement; on the contrary, it can in fact amount to the vindication of the scientific quality of the concept of agency. In this sense, reductionism is the only compatibilism of agency and physical causal-closure.

Acknowledgements This commentary elaborates on my commentary on Horgan, I presented at the conference Mental causation, externalism, and self-knowledge (2005, University of Tübingen). My thanks to the organizers of that conference, and the participants, especially Michael Esfeld, Jens Harbecke, Vera Hoffmann, Terry Horgan and Georg Sparber. The work on this commentary has been supported by the Swiss National Science Foundation (SNF), grant nr. 100011-105218/1

\section{References:}

Bickle, J. (1998). Psychoneural reduction: The new wave. Cambridge (MA): MIT Press.

Chalmers, D. J. (1996). The conscious mind. In search of a fundamental theory. Oxford: Oxford University Press.

Endicott, R. (1998). Collapse of the new wave. Journal of Philosophy, 95, 53-72.

Esfeld, M., \& Sachse, C. (2007). Theory reduction by means of functional sub-types. International Studies in the Philosophy of Science, 21(1), 1-17.

Heil, J. (2003). From an ontological point of view. Oxford: Clarendon Press.

Horgan, T. (2001). Multiple reference, multiple realization, and the reduction of mind. In F. Siebelt \& B. Preyer (Eds.), Reality and Humean supervenience: Essays on the philosophy of David Lewis (pp. 205-221). Rowman \& Littlefield.

Kim, J. (2005). Physicalism, or something near enough. Princeton: Princeton University Press.

Sachse, C. (2007). Reductionism in the philosophy of science. Frankfurt (Main): Ontos-Verlag. 